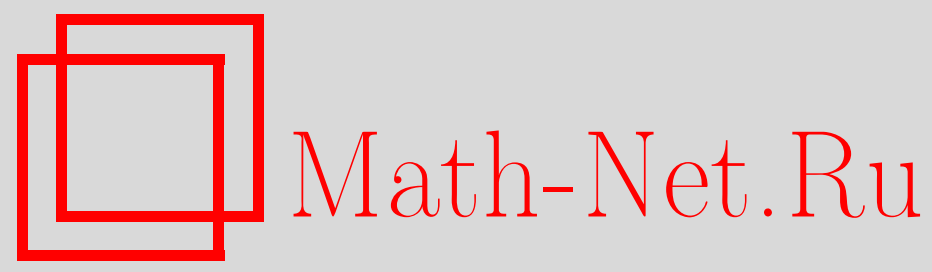

В. Н. Потапов, Д. С. Кротов, О числе $n$-арных квазигрупп конечного порядка, Дискрет. матем., 2012, том 24, выпуск 1, 60-69

DOI: https://doi.org/10.4213/dm1172

Использование Общероссийского математического портала Math-Net.Ru подразумевает, что вы прочитали и согласны с пользовательским соглашением http://www . mathnet.ru/rus/agreement

Параметры загрузки:

IP: 34.227 .88 .159

26 апреля 2023 г., $13: 28: 38$ 


\title{
О числе $n$-арных квазигрупп конечного порядка
}

\author{
() 2012 г. В. Н. Потапов, Д. С. Кротов
}

Пусть $Q(n, k)$ - число $n$-арных квазигрупп порядка $k$. Получена рекуррентная формула для чисел $Q(n, 4)$. Доказано, что при любых $n \geqslant 2$ и $k \geqslant 5$ справедливы неравенства

$$
((k-3) / 2)^{n / 2}((k-1) / 2)^{n / 2}<\log _{2} Q(n, k) \leqslant c_{k}(k-2)^{n},
$$

где $c_{k}$ не зависит от $n$. Таким образом, верхняя асимптотическая граница для чисел $Q(n, k)$ улучшена при любых $k \geqslant 5$, нижняя - при нечетных $k \geqslant 7$.

Работа выполнена при поддержке ФЦП «Научные и научно-педагогические кадры инновационной России» на 2009-2013 гг. (гос. контракт №02.740.11.0429) и Российского фонда фундаментальных исследований, проекты 08-01-00671, 08-01-00673, 10-01-00616.

\section{1. Введение}

Алгебраическая система из множества $\Sigma$ мощности $|\Sigma|=k$ и $n$-арной операции $f$ : $\Sigma^{n} \rightarrow \Sigma$ называется $n$-арной квазигруппой порядка $k$, если унарная операция, полученная фиксацией любых $n-1$ аргументов операции $f$ любыми значениями из $\Sigma$, всегда является биекцией. Принято называть $n$-арной квазигруппой порядка $k$ или $n$-квазигруппой порядка $k$ также и соответствующую функцию $f$ (таблица значений такой функции известна под названием латинский гиперкуб, в случае $n=2-$ латинский квадрат).

Зафиксируем множество элементов $\Sigma=\{0,1, \ldots, k-1\}$. Обозначим через $Q(n, k)$ число различных $n$-арных квазигрупп порядка $k$ (при фиксированном $\Sigma$ ). Иногда под числом квазигрупп подразумевают число попарно неизоморфных квазигрупп. Известно, что для каждого $n$ существует только две $n$-арных квазигруппы порядка 2 . Имеется $Q(n, 3)=3 \cdot 2^{n}$ различных $n$-арных квазигрупп порядка 3 , которые составляют единственный класс эквивалентности. В [1] доказано асимптотическое равенство

$$
Q(n, 4)=3^{n+1} 2^{2^{n}+1}(1+o(1))
$$

при $n \rightarrow \infty$. В настоящей работе (в разделе 4) предложен рекуррентный способ вычисления чисел $Q(n, 4)$, выписаны первые 8 значений этой величины. Ранее были известны только пять первых значений $Q(n, 4)$, кроме того, известны числа $Q(n, 5)$ и $Q(n, 6)$ для $n \leqslant 5$ и $n \leqslant 3$ соответственно (см. [9]) и число $Q(2, k)$ при $k \leqslant 11$ (см. [8] и библиографию в ней).

Асимптотика числа и даже логарифма числа (и даже логарифма логарифма числа) $n$-арных квазигрупп порядков, больших 4, неизвестна. В [7] получены следующие нижние 
оценки:

$$
\begin{array}{ll}
Q(n, 5) \geqslant 2^{3^{n / 3}-c}, & c<0,072 ; \\
Q(n, k) \geqslant 2^{(k / 2)^{n}}, & k \text { четно; } \\
Q(n, k) \geqslant 2^{n(k / 3)^{n}}, & k \text { кратно трем; } \\
Q(n, k) \geqslant 2^{\frac{3}{2}\lfloor k / 3\rfloor^{n}} . &
\end{array}
$$

В [4] для числа $n$-арных квазигрупп порядка $k$ была предложена верхняя оценка

$$
Q(n, k) \leqslant 3^{(k-2)^{n}} 2^{n(k-2)^{n-1}} .
$$

В разделе 2 настоящей статьи усилена верхняя оценка числа $n$-арных квазигрупп конечного порядка и в раздел 3 усилена нижняя оценка числа $n$-арных квазигрупп нечетного порядка

$$
\left(\frac{k-3}{2}\right)^{n / 2}\left(\frac{k-1}{2}\right)^{n / 2} \log _{2} Q(n, k) \leqslant c_{k}(k-2)^{n},
$$

где $c_{k}$ не зависит от $n$, точнее,

$$
c_{k}=\frac{\log _{2} k !}{k-2}+\frac{k}{k-4} .
$$

\section{2. Верхняя оценка}

Будем говорить, что множество $M \subseteq \Sigma^{n}$ удовлетворяет свойству (А), если для любого элемента $\bar{x}$ из $M$ и каждой позиции $i=1, \ldots, n$ найдется другой элемент $\bar{y}$ из $M$, отличающийся от $\bar{x}$ только в позиции $i$. По индукции легко получить следующее утверждение.

Предложение 1. Любое непустое подмножество $C \subseteq \Sigma^{n}$, удовлетворяющее свойству (А), имеет мощность не менее $2^{n}$.

Частичной $n$-арной квазигруппой порядка $|\Sigma|$ называется функция $g: \Omega \rightarrow \Sigma$, где $\Omega \subset \Sigma^{n}$, удовлетворяющая следующему свойству: $g(\bar{x}) \neq g(\bar{y})$ для любых двух наборов $\bar{x}, \bar{y} \in \Omega$, отличающихся ровно в одной позиции. Будем говорить, что $n$-арная квазигруппа $f: \Sigma^{n} \rightarrow \Sigma$ является продолжением частичной $n$-арной квазигруппы $g: \Omega \rightarrow \Sigma$, где $\Omega \subset \Sigma^{n}$, если $\left.f\right|_{\Omega} \equiv g$.

Лемма 1. Пусть $|\Sigma|=k, B=\Sigma \backslash\{a, b\}, k \geqslant 3, a, b \in \Sigma$. Тогда частичная n-арная квазигруппа $g: \Sigma^{n-1} \times B \rightarrow \Sigma$ имеет не более чем $2^{(k / 2)^{n-1}}$ различных продолжений.

Доказательство. Пусть $P-$ множество неупорядоченных пар элементов множества $\Sigma$. Рассмотрим частичную $n$-арную квазигруппу $g: \Sigma^{n-1} \times B \rightarrow \Sigma$. Определим функцию $G: \Sigma^{n-1} \rightarrow P$ равенством

$$
G(\bar{x})=\Sigma \backslash\{g(\bar{x} c): c \in \Sigma \backslash\{a, b\}\} .
$$

Определим граф $\Gamma=\left\langle\Sigma^{n-1}, E\right\rangle$, в котором две вершины $\bar{x}$ и $\bar{y}$ соединены ребром тогда и только тогда, когда наборы $\bar{x}$ и $\bar{y}$ отличаются только в одной позиции и $G(\bar{x}) \cap G(\bar{y}) \neq \varnothing$. Нетрудно видеть, что компоненты связности графа Г удовлетворяют свойству (А). 
Пусть $n$-арные квазигруппы $f_{1}$ и $f_{2}$ являются продолжениями частичной $n$-арной квазигруппы $g$. Нетрудно видеть, что

$$
\left\{f_{1}(\bar{x} a), f_{1}(\bar{x} b)\right\}=G(\bar{x})
$$

для любого $\bar{x} \in \Sigma^{n-1}$, причем, если $f_{1}(\bar{x} a)=f_{2}(\bar{x} a)$, то продолжения $f_{1}$ и $f_{2}$ совпадают на всей компоненте связности графа $\Gamma$, содержащей вершину $\bar{x} \in \Sigma^{n-1}$. Таким образом, для однозначного определения продолжения частичной $n$-арной квазигруппы $g$ достаточно зафиксировать одно из двух возможных значений в каждой компоненте связности графа Г. Из предложения 1 следует, что каждая компонента связности имеет мощность не менее $2^{n-1}$. Тогда число компонент связности графа $\Gamma$ не превосходит $(k / 2)^{n-1}$. Значит, $g$ имеет не более $2^{(k / 2)^{n-1}}$ продолжений.

Теорема 1. Если $k \geqslant 5 u n \geqslant 2$, то

$$
Q(n, k) \leqslant 2^{c_{k}(k-2)^{n}},
$$

где

$$
c_{k}=\frac{\log _{2} k !}{k-2}+\frac{k}{k-4} .
$$

Доказательство. Число частичных $n$-арных квазигрупп $g: \Sigma^{n-1} \times B \rightarrow \Sigma$, где $|\Sigma|=k$, $B=\Sigma \backslash\{a, b\}$, не превосходит $Q(n, k)^{k-2}$. Из леммы 1 следует неравенство

$$
Q(n+1, k) \leqslant Q(n, k)^{k-2} 2^{(k / 2)^{n}} .
$$

Введем обозначение

$$
\alpha_{n}=\frac{\log _{2} Q(n, k)}{(k-2)^{n}} .
$$

Тогда из неравенства (1) следует, что

$$
\alpha_{n+1} \leqslant \alpha_{n}+\left(\frac{k}{2(k-2)}\right)^{n} .
$$

Поскольку

$$
\begin{aligned}
\alpha_{1} & =\frac{\log _{2} k !}{k-2}, \\
\sum_{n=1}^{\infty}\left(\frac{k}{2(k-2)}\right)^{n} & =\frac{k}{k-4},
\end{aligned}
$$

справедливо неравенство

$$
\alpha_{n} \leqslant \frac{\log _{2} k !}{k-2}+\frac{k}{k-4}
$$

\section{3. Нижняя оценка}

Пусть $a$ и $b$ - два различных элемента из $\Sigma$. Будем называть $\{a, b\}$-компонентой $n$-арной квазигруппы $f$ такое множество $S \subset \Sigma^{n}$, что

(1) $f(S)=\{a, b\}$ и 
(2) функция

$$
g(\bar{x})= \begin{cases}f(\bar{x}) & \text { при } \bar{x} \notin S, \\ b & \text { при } \bar{x} \in S \text { и } f(\bar{x})=a, \\ a & \text { при } \bar{x} \in S \text { и } f(\bar{x})=b\end{cases}
$$

также является $n$-арной квазигруппой.

В этом случае будем говорить, что $g$ получена из $f$ свитчингом компоненты $S$. Заметим, что в определении $\{a, b\}$-компоненты условие 2 можно заменить свойством (А) из предыдущего раздела. Очевидно, что свитчинг непересекающихся компонент можно производить независимо.

Предложение 2. Пусть $S u S^{\prime}$ - непересекаюшиеся $\{a, b\}-u\{c, d\}$-компоненты n-арной квазигруппь $f$ соответственно, и п-арная квазигруппа $g$ получена из $f$ свитчингом компоненты $S$. Тогда $S^{\prime}$ также является $\{c, d\}$-компонентой квазигруппь $g$.

Следующее предложение нетрудно получить из определения $\{a, b\}$-компоненты, аналогичное утверждение имеется в [7].

Предложение 3. Пусть множество $C=\left\{c_{1}, d_{1}\right\} \times\left\{c_{2}, d_{2}\right\}$ является $\{a, b\}$-компонентой 2-квазигруппь $g$. Пусть множество $C_{i}$ является $\left\{c_{i}, d_{i}\right\}$-компонентой $n_{i}$-арной квазигруппы $q_{i}$ при $i=1,2$. Тогда множество $C_{1} \times C_{2}$ является $\{a, b\}$-компонентой $\left(n_{1}+n_{2}\right)$ арной квазигруппь $f$, где

$$
f\left(\bar{x}_{1}, \bar{x}_{2}\right) \equiv g\left(q_{1}\left(\bar{x}_{1}\right), q_{2}\left(\bar{x}_{2}\right)\right) .
$$

Назовем 2-квазигруппу $\varphi: \Sigma \rightarrow \Sigma$ идемпотентной, если $\varphi(x, x)=x$ для любого $x \in \Sigma$. Известно (см., например, [2]), что верно следующее утверждение.

Предложение 4. Для любого $m \geqslant 3$ имеется идемпотентная 2-квазигруппа порядка $m$.

В следующем предложении приведена конструкция 2-квазигрупп, которая будет использована при доказательстве нижней оценки числа $n$-арных квазигрупп нечетного порядка.

Предложение 5. Для любого $m \geqslant 3$ найдется 2-квазигруппа $\psi$ порядка $2 m+1$, имеющая $m\{2 i, 2 i+1\}$-компонент для каждого $i \in\{0, \ldots, m-1\}$, причем все, кроме одной, $\{2 i, 2 i+1\}$-компоненты имеют вид $\{2 j, 2 j+1\} \times\{2 l, 2 l+1\}$.

Доказательство. По предложению 4, найдется идемпотентная 2-квазигруппа $\varphi_{m}$ порядка $m$. Для любых $a, b \in\{0, \ldots, m-1\}, a \neq b$, и $\delta, \sigma \in\{0,1\}$ положим

$$
\begin{aligned}
\psi(2 a+\delta, 2 b+\sigma) & =2 \varphi_{m}(a, b)+(\delta+\sigma \bmod 2) ; \\
\psi(2 a+\delta, 2 a+\delta) & =2 a+1-\delta ; \\
\psi(2 a+\delta, 2 a+1-\delta) & =k-1 ; \\
\psi(k-1,2 a+\delta) & =\psi(2 a+\delta, k-1)=2 a+\delta ; \\
\psi(k-1, k-1) & =k-1 .
\end{aligned}
$$

Непосредственная проверка показывает, что $\psi$ есть 2-квазигруппа, обладающая требуемыми свойствами.

Ниже приведен пример таблиц значений 2-квазигруппы $\varphi_{4}$ и соответствующей $\psi$ : 


$\varphi_{4}: \quad$\begin{tabular}{llll|}
0 & 2 & 3 & 1 \\
3 & 1 & 0 & 2 \\
1 & 3 & 2 & 0 \\
2 & 0 & 1 & 3 \\
\hline
\end{tabular}

\begin{tabular}{ll|ll|ll|ll|l|}
\hline 1 & 8 & 4 & 5 & 6 & 7 & 2 & 3 & 0 \\
8 & 0 & 5 & 4 & 7 & 6 & 3 & 2 & 1 \\
\hline 6 & 7 & 3 & 8 & 0 & 1 & 4 & 5 & 2 \\
7 & 6 & 8 & 2 & 1 & 0 & 5 & 4 & 3 \\
\hline 2 & 3 & 6 & 7 & 5 & 8 & 0 & 1 & 4 \\
3 & 2 & 7 & 6 & 8 & 4 & 1 & 0 & 5 \\
\cline { 1 - 6 } 4 & 5 & 0 & 1 & 2 & 3 & 7 & 8 & 6 \\
5 & 4 & 1 & 0 & 3 & 2 & 8 & 6 & 7 \\
\hline 0 & 1 & 2 & 3 & 4 & 5 & 6 & 7 & 8 \\
\hline
\end{tabular}

Из предложения 1 нетрудно заключить, что 2-квазигруппа нечетного порядка $k$, построенная в предложении 5, имеет максимальное число непересекающихся компонент среди всех 2-квазигрупп порядка $k$.

Теорема 2. Если $k \geqslant 5$ - нечетное число и $n \geqslant 2$, то

$$
Q(n, k) \geqslant 2^{((k-3) / 2)^{\lfloor(n-1) / 2\rfloor}((k-1) / 2)^{\lceil(n+1) 2\rceil}}>2^{((k-3) / 2)^{n / 2}((k-1) / 2)^{n / 2}} .
$$

Доказательство. Пусть $\psi-2$-квазигруппа порядка $k$, построенная в предложении 5. Определим рекуррентно $n$-арную квазигруппу $\Psi^{n}$ равенствами

$$
\begin{aligned}
\Psi^{2} & \equiv \psi ; \\
\Psi^{2 m+1}(\bar{x}, y) & =\psi\left(\Psi^{2 m}(\bar{x}), y\right) ; \\
\Psi^{2 m+2}(\bar{x}, y, z) & =\psi\left(\Psi^{2 m}(\bar{x}), \psi(y, z)\right) .
\end{aligned}
$$

Обозначим через $\alpha_{n}$ число $\{2 i, 2 i+1\}$-компонент $n$-арной квазигруппы $\Psi^{n}$, где $i \in\{0, \ldots,(k-3) / 2\}$. Из предложений 3 и 5 следуют соотношения $\alpha_{2}=(k-1) / 2$, $\alpha_{2 m+1} \geqslant \alpha_{2 m}(k-3) / 2, \alpha_{2 m+2} \geqslant \alpha_{2 m}((k-3) / 2)((k-1) / 2)$. Тогда

$$
\alpha_{2 m} \geqslant((k-3) 2)^{m-1}\left((k-1) / 2^{m}, \quad \alpha_{2 m+1} \geqslant((k-3) / 2)^{m}((k-1) / 2)^{m} .\right.
$$

Поскольку $\{2 i, 2 i+1\}$-компоненты при различных $i$ не пересекаются, всего непересекающихся компонент не меньше, чем $(k-1) \alpha_{n} / 2$. Из предложения 2 следует, что из $n$-арной квазигруппы $\Psi^{n}$ свитчингами непересекающихся компонент можно получить требуемое число различных $n$-арных квазигрупп порядка $k$.

\section{4. Число $n$-арных квазигрупп порядка 4}

Введем обозначение $[n]=\{1, \ldots, n\} ; n$-арная квазигруппа $f$ называется $n$-арной лупой, если существует такой элемент $e \in \Sigma$, называемый единичным, что для всех $i \in[n]$ и $a \in \Sigma$ имеет место равенство $f(e \ldots e a e \ldots e)=a$. Далее мы всегда будем подразумевать, что 0 является единичным элементом $n$-арной лупы (в общем случае могут быть и другие единичные элементы). Особо отметим, что данное соглашение существенно в интерпретации понятия числа $n$-арных луп. В частности, справедлив следующий простой и хорошо известный факт.

Предложение 6. Пусть $Q^{\prime}(n, k)$ - число n-арньх луп порядка $k$. Тогда

$$
Q(n, k)=k((k-1) !)^{n} Q^{\prime}(n, k) .
$$


Разделимой (приводимой) называется $n$-арная квазигруппа $f$, если найдутся целое число $m, 2 \leqslant m<n,(n-m+1)$-арная квазигруппа $h, m$-арная квазигруппа $g$ и перестановка $\sigma:[n] \rightarrow[n]$, такие, что

$$
f\left(x_{1}, \ldots, x_{n}\right) \equiv h\left(g\left(x_{\sigma(1)}, \ldots, x_{\sigma(m)}\right), x_{\sigma(m+1)}, \ldots, x_{\sigma(n)}\right) .
$$

В дальнейшем будем предполагать, что $\Sigma=\{0,1,2,3\}$, то есть речь пойдет только об $n$-арных квазигруппах порядка 4. Известно (см., например, [2]), что имеется ровно четыре бинарные лупы порядка 4 (одна изоморфна группе $Z_{2} \times Z_{2}$ и три изоморфны группе $Z_{4}$ ).

Следующее утверждение является прямым следствием теоремы из [3].

Лемма 2. Для разделимой $n$-арной лупь $f$ порядка 4 справедливо одно и только одно из двух представлений:

$$
f(\bar{x})=q_{0}\left(q_{1}\left(\tilde{x}_{1}\right), \ldots, q_{m}\left(\tilde{x}_{m}\right)\right),
$$

где $q_{j}$ есть $n_{j}$-арные лупы при $j=1, \ldots, m, q_{0}$ есть неразделимая $m$-арная лупа, $m \geqslant 3$, $\tilde{x}_{j}$ - некоторые наборы переменных $x_{i}, i \in I_{j}$, где $\left\{I_{j}\right\}-$ разбиение множества $[n]$, причем в данном представлении разбиение $\left\{I_{j}\right\}$ единственно, $u$

$$
f(\bar{x})=q_{1}\left(\tilde{x}_{1}\right) * \ldots * q_{k}\left(\tilde{x}_{k}\right),
$$

где * есть бинарная операщия в одной из 4 луп, $q_{j}$ есть $n_{j}$-арныле лупь при $j, 1 \leqslant j \leqslant k$, непредставимые в виде $q_{j}\left(\tilde{x}_{j}\right)=q^{\prime}\left(\tilde{x}_{j}^{\prime}\right) * q^{\prime \prime}\left(\tilde{x}_{j}^{\prime \prime}\right), \tilde{x}_{j}-$ некоторые наборы переменных $x_{i}$, $i \in I_{j}$, где $\left\{I_{j}\right\}$ - разбиение множества $[n]$, причем в данном представлении разбиение $\left\{I_{j}\right\}$ единственно.

Корневой операцией $n$-арной квазигруппы $f$ будем называть $m$-арную квазигруппу $q_{0}$, если имеет место представление (2), и бинарную операцию $*$, если имеет место представление (3).

Простой комбинаторный подсчет показывает, что число $F_{\bar{j}, \bar{k}}$ различных разбиений множества $[n]$ на $k$ подмножеств, из которых $k_{i}$ подмножеств имеет мощность $j_{i}$, $1 \leqslant i \leqslant t, 0<j_{1}<\ldots<j_{t}$, удовлетворяет равенству

$$
F_{\bar{\jmath}, \bar{k}}=\frac{n !}{\left(j_{1} !\right)^{k_{1}} \ldots\left(j_{t} !\right)^{k_{t}}} \frac{1}{k_{1} ! \ldots k_{t} !},
$$

где

$$
\begin{aligned}
k_{1}+k_{2}+\ldots+k_{t} & =k, \\
k_{1} j_{1}+k_{2} j_{2}+\ldots+k_{t} j_{t} & =n .
\end{aligned}
$$

Пусть $f: \Sigma^{n} \rightarrow \Sigma-n$-арная квазигруппа, определим множество

$$
S_{a, b}(f) \triangleq\left\{\bar{x} \in \Sigma^{n}: f(\bar{x}) \in\{a, b\}\right\} .
$$

Назовем $n$-арную лупу $f a$-полулинейной, где $a \in\{1,2,3\}$ если характеристическая функция $\chi_{S_{0, a}(f)}$ множества $S=S_{0, a}(f)$ имеет вид

$$
\chi_{S_{0, a}(f)}\left(x_{1}, \ldots, x_{n}\right) \equiv \sum_{i=1}^{n} \chi_{\{0, a\}}\left(x_{i}\right) \bmod 2 .
$$

Будем называть линейной $n$-арную квазигруппу $f$, если она одновременно является $a$-полулинейной и $b$-полулинейной, где $a \neq b, a, b \in\{1,2,3\}$. Непосредственной проверкой нетрудно убедиться, что справедливо следующее утверждение. 
Предложение 7. Из четырех бинарных луп порядка 4 одна (изоморфная группе $Z_{2} \times Z_{2}$ ) является линейной, а три остальных - 1-, 2- и 3-полулинейными соответственно.

Справедливо следующее утверждение (см. [1]).

Предложение 8. Линейная n-арная лупа единственна и является одновременно 1-, 2- $u$ 3-полулинейной.

Нетрудно видеть (см. также [1]), что справедливо следующее утверждение.

Предложение 9. Пусть $f$ - разделимая а-полулинейная $n$-арная лупа, тогда $f$ можно представить как суперпозищию а-полулинейных луп вида (2) или (3).

Обозначим через $l_{n}^{a}$ мощность множества $a$-полулинейных $n$-арных луп и через $l_{n}-$ мощность множества полулинейных $n$-арных луп.

Как было установлено в [1], мощность множества всех $n$-арных луп асимптотически совпадает с мощностью множества полулинейных $n$-арных луп, которая легко вычисляется.

Лемма 3 ([1]). Справедливы равенства

$$
l_{n}=3 \cdot 2^{2^{n}-n-1}-2, \quad l_{n}^{a}=2^{2^{n}-n-1}, \quad a \in\{1,2,3\} .
$$

В [6] получено описание $n$-арных квазигрупп порядка 4 в определенных выше терминах, а именно, доказана следующая теорема.

Теорема 3. Каждая n-арная лупа порядка 4 является разделимой или полулинейной.

На этом описании по-существу основывается вывод рекуррентной формулы для числа $n$-арных луп (и квазигрупп) порядка 4.

Введем следующие обозначения:

$v_{n}$ - число $n$-арных луп (порядка 4);

$r_{n}^{*}$ - число разделимых $n$-арных луп с бинарной корневой операцией $*$;

$r_{n}^{0}$ - число разделимых $n$-арных луп с корневой операцией арности, большей либо равной 3 ;

$r_{n}^{a *}$ - число разделимых $a$-полулинейных $n$-арных луп с $a$-полулинейной бинарной корневой операцией $*$;

$r_{n}^{a 0}$ - число разделимых $a$-полулинейных $n$-арных луп с корневой операцией арности, большей либо равной 3 ;

$p_{n}^{a}$ - число неразделимых $a$-полулинейных $n$-арных луп;

$p_{n}$ - число неразделимых $n$-арных луп. 
Из леммы 2 и предложения 9 вытекают следующие соотношения:

$$
\begin{aligned}
r_{n}^{a *} & =\sum_{i=2}^{n} \sum_{\bar{\jmath}, \bar{k}} F_{\bar{\jmath}, \bar{k}}\left(l_{j_{1}}^{a}-r_{j_{1}}^{a *}\right)^{k_{1}} \cdots\left(l_{j_{t}}^{a}-r_{j_{t}}^{a *}\right)^{k_{t}}, \\
r_{n}^{*} & =\sum_{i=2}^{n} \sum_{\bar{\jmath}, \bar{k}} F_{\bar{\jmath}, \bar{k}}\left(v_{j_{1}}-r_{j_{1}}^{*}\right)^{k_{1}} \cdots\left(v_{j_{t}}-r_{j_{t}}^{*}\right)^{k_{t}}, \\
r_{n}^{a 0}= & \sum_{i=3}^{n-1} p_{i}^{a} \sum_{\bar{\jmath}, \bar{k}} F_{\bar{\jmath}, \bar{k}}\left(l_{j_{1}}^{a}\right)^{k_{1}} \cdots\left(l_{j_{t}}^{a}\right)^{k_{t}}, \\
r_{n}^{0}= & \sum_{i=3}^{n-1} p_{i} \sum_{\bar{j}, \bar{k}} F_{\bar{j}, \bar{k}}\left(v_{j_{1}}\right)^{k_{1}} \cdots\left(v_{j_{t}}\right)^{k_{t}},
\end{aligned}
$$

где вторая сумма берется по наборам $\bar{k}=\left(k_{1}, \ldots, k_{t}\right)$ и $\bar{j}=\left(j_{1}, \ldots, j_{t}\right)$ положительных целых чисел, удовлетворяющих равенствам

$$
\begin{aligned}
k_{1}+\ldots+k_{t} & =i, \\
k_{1} j_{1}+k_{2} j_{2}+\ldots+k_{t} j_{t} & =n
\end{aligned}
$$

и неравенствам $j_{1}<\ldots<j_{t}$. Из теоремы 3 и предложения 8 вытекают соотношения

$$
\begin{aligned}
& v_{n}=p_{n}+r_{n}^{0}+4 r_{n}^{*}, \\
& p_{n}^{a}=l_{n}^{a}-r_{n}^{a 0}-2 r_{n}^{a *}, \\
& p_{n}=3 p_{n}^{a} .
\end{aligned}
$$

Из леммы 3 следует, что

$$
l_{n}^{a}=2^{2^{n}-n-1}, \quad a \in\{1,2,3\} .
$$

Из предложения 7 получаем начальные значения для перечисленных выше величин:

$$
\begin{aligned}
r_{2}^{a *} & =2, \\
r_{2}^{*} & =4, \\
r_{2}^{a 0}=r_{2}^{0} & =0 .
\end{aligned}
$$

Нетрудно видеть, что приведенные выше равенства и предложение 6 обеспечивают рекуррентный способ вычисления числа $n$-арных квазигрупп порядка 4.

Наконец, выпишем первые восемь значений величины $Q^{\prime}(n, 4)$ :

1 ,

4 ,

64 ,

7132 ,

201538000 ,

432345572694417712 ,

3987683987354747642922773353963277968 ,

678469272874899582559986240285280710364867063489779510427038722229750276832 ,

и $Q(n, 4)$ : 
24 ,

576 ,

55296 ,

36972288 ,

6268637952000 ,

80686060158523011084288 ,

4465185218736554544676917926460256725000192 ,

4558271384916189349044295395852008182480786230841798008741684281906576963885826048.

\section{5. Заключение}

В заключение скажем несколько слов о связи тематики настоящей статьи с известным понятием латинского трэйда. Частичная $n$-арная квазигруппа $t: \Omega \rightarrow \Sigma, \Omega \subset \Sigma^{n}$, называется многомерным латинским трэйдом, далее просто трэйдом, если существует другая частичная $n$-арная квазигруппа $t^{\prime}: \Omega \rightarrow \Sigma$ такая, что

(1) $t(\bar{x}) \neq t^{\prime}(\bar{x})$ для всех $\bar{x} \in \Omega$;

(2) для любого $i, i=1, \ldots, n$, и любых допустимых значений $x_{1}, \ldots, x_{n}$ множества $\left\{t\left(x_{1}, \ldots, x_{i-1}, y, x_{i-1}, \ldots, x_{n}\right) \mid y \in \Sigma\right\}$ и $\left\{t^{\prime}\left(x_{1}, \ldots, x_{i-1}, y, x_{i-1}, \ldots, x_{n}\right) \mid y \in \Sigma\right\}$ совпадают.

В этом случае пара $\left(t, t^{\prime}\right)$ называется битрэйдом (в зависимости от контекста, битрэйд рассматривают как упорядоченную, либо как неупорядоченную пару), а трэйд $t^{\prime}$ называется партнером трэйда $t$. Исследованию битрэйдов в случае $n=2$ (латинских битрэйдов) уделяется значительное внимание, см. обзор [5].

Будем говорить, что $n$-арная квазигруппа $f$ содержит трэйд $t$, если $t=\left.f\right|_{\Omega}$ для некоторого $\Omega$. При этом из определений следует, что замена значений $f$ на множестве $\Omega$ значениями партнера $t^{\prime}$ трэйда $t$ приводит к другой $n$-арной квазигруппе. Трэйды $t=\left.f\right|_{\Omega}$ и $s=\left.f\right|_{\Theta}$ назовем независимыми, если их носители (области определения) $\Omega$ и $\Theta$ не пересекаются. Максимальное число попарно независимых трэйдов, которые содержит $n$-арная квазигруппа $f$, назовем ее трэйдовым числом $\operatorname{trd}(f)$. Максимум $\operatorname{trd}(f)$ по всем $n$-арным квазигруппам $f$ порядка $k$ обозначим через $\operatorname{Trd}(n, k)$. Поскольку независимые трэйды в $n$-арной квазигруппе можно независимо заменять на партнеров, число $Q(n, k)$ различных $n$-арных квазигрупп порядка $k$ удовлетворяет неравенству

$$
Q(n, k) \geqslant 2^{\operatorname{Trd}(n, k)} .
$$

Легко понять, что нижняя оценка в разделе 3 (как и все оценки в [7]) получена именно таким образом: $\{a, b\}$-компонента по определению является носителем некоторого трэйда. Поскольку носитель трэйда обладает свойством (А), из предложения 1 вытекает соотношение

$$
\operatorname{Trd}(n, k) \leqslant k^{n} / 2^{n}=2^{\left(\log _{2} k-1\right) n},
$$

причем для четных $k$ легко доказать равенство. Для нечетных $k$ из результатов раздела 3 следует оценка

$$
\operatorname{Trd}(n, k) \geqslant 2^{c(k) n},
$$


где

$$
c(k) \underset{k \rightarrow \infty}{\longrightarrow} \log _{2} k-1 .
$$

Однако для фиксированных $k$, в частности, для малых значений $5,7, \ldots$ вопрос об асимптотическом поведении величины $\operatorname{Trd}(n, k)$ остается открытым.

Проблема 1. При $n \rightarrow \infty$ вычислить асимптотику логарифма и асимптотику величины $\operatorname{Trd}(n, k)$ для нечетных $k \geqslant 5$.

Другой вопрос состоит в том, насколько оценка (6) близка к истинной. Для порядка 4 оценка (6) асимптотически точна после логарифмирования. Для большего фиксированного порядка асимптотика двукратного логарифма величины $Q(n, k)$ неизвестна. Кажется естественным предположить, что асимптотика двукратного логарифма величины $Q(n, k)$ и логарифма величины $\operatorname{Trd}(n, k)$ совпадают.

Проблема 2. Верно ли, что

$$
\lim _{n \rightarrow \infty}\left(\frac{\log _{2} \log _{2} Q(n, k)}{n}\right)=\lim _{n \rightarrow \infty}\left(\frac{\log _{2} \operatorname{Trd}(n, k)}{n}\right) ?
$$

В частности, верно ли, что

$$
\lim _{n \rightarrow \infty}\left(\frac{\log _{2} \log _{2} Q(n, k)}{n}\right) \leqslant \log _{2} k-1 ?
$$

Существование этих пределов также не доказано.

\section{Список литературы}

1. Потапов В. Н., Асимптотика числа $n$-квазигрупп порядка 4. Сибирский матем. журнал (2006) 47, №4, 873-887.

2. Белоусов В. Д., Основы теории квазигрупп. Наука, Москва, 1967.

3. Черемушкин А. В., Каноническая декомпозищия n-арных квазигрупп. Штиинца, Кишинев, 1988.

4. Потапов В. Н., Верхняя оценка числа $n$-квазигрупп конечного порядка. В сб.: Tpyды XVII международной школь-семинара «Синтез и сложность управляющих систем». Новосибирск, 2008, c. $135-137$.

5. Cavenagh N. J., The theory and application of latin bitrades: A survey. Mathematica Slovaca (2008) 58, 691-718.

6. Krotov D. S., Potapov V. N., n-ary quasigroups of order 4. SIAM J. Discrete Math. (2009) 23, 561-570.

7. Krotov D. S., Potapov V. N., Sokolova P. V., On reconstructing reducible $n$-ary quasigroups and switching subquasigroups. Quasigroups Relat. Syst. (2008) 16, 55-67.

8. McKay B. D., Wanless I. M., On the number of Latin squares. Ann. Comb. (2005) 9, 335-344.

9. McKay B. D., Wanless I. M., A census of small Latin hypercubes. SIAM J. Discrete Math. (2008) 22, 719-736. 